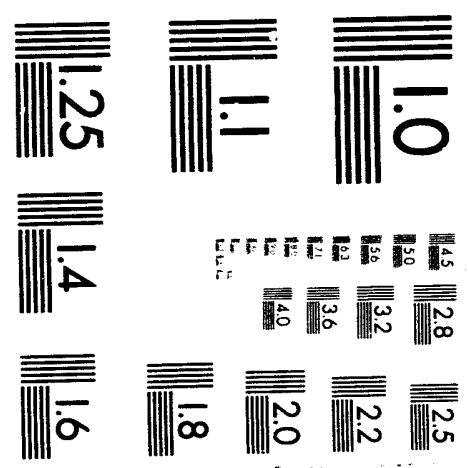



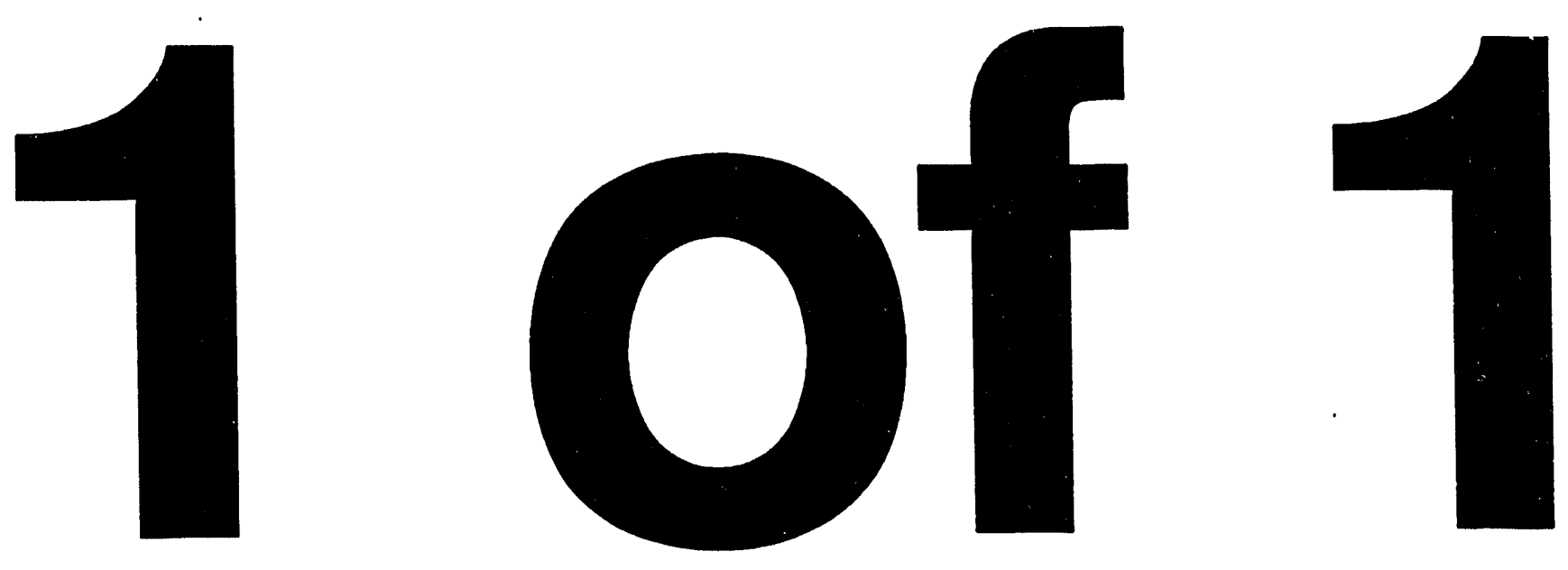


\title{
RECENT PROGRESS IN NONPERTURBATIVE ELECTROMAGNETIC LEPTON-PAIR PRODUCTION WITH CAPTURE IN RELATIVISTIC HEAVY-ION COLLISIONS
}

\author{
J. C. Wells ${ }^{1,2}$, V. E. Oberacker ${ }^{2}$, M. R. Strayer ${ }^{1}$, and A. S. Umar ${ }^{2}$ \\ ${ }^{1}$ Oak Ridge National Laboratory \\ Oak Ridge, TN 37831-6373 \\ ${ }^{2}$ Vanderbilt University \\ Nashville, TN 37235
}

to be published in

Proceedings International Advanced Courses on Hot and Dense Nuclear Matter

Bodrum, Turkey

September 26-October 9, 1993

DISCLAIMER

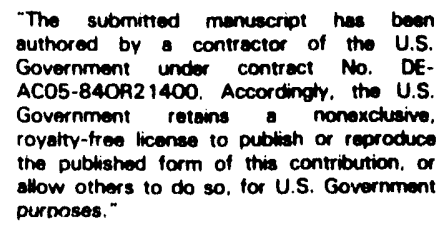

This report was prepared as an account of work sponsored by an agency of the United States Government. Neither the United States Government nor any agency thereof, nor any of their employees, makes any warranty, express or implied, or assumes any legal liability or responsibility for the accuracy, completeness, or usefulness of any information, apparatus, product, or process disclosed, or represents that its use would not infringe privately owned rights. Reference herein to any specific commercial product, process, or service by trade name, trademark, manufacturer, or otherwise does not necessarily constitute or imply its endorsement, recommendation, or favoring by the United States Government or any agency thereof. The views and opinions of authors expressed herein do not necessarily state or reflect those of the United States Government or any agency thereof. 


\title{
RECENT PROGRESS IN NONPERTURBATIVE ELECTROMAGNETIC LEPTON-PAIR PRO- DUCTION WITH CAPTURE IN RELATIVISTIC HEAVY-ION COLLISIONS
}

\author{
J. C. Wells ${ }^{1,2}$, V. E. Oberacker ${ }^{1,2}$, M. R. Strayer ${ }^{1}$ A. S. Umar ${ }^{1,2}$ \\ ${ }^{1}$ Center for Computationally Intensive Physics, \\ Physics Division, Oak Ridge National Laboratory, \\ Oak Ridge, TN 37831-6373 \\ 2 Department of Physics \& Astronomy, \\ Vanderbilt University, Nashville, TN 37235
}

\section{INTRODUCTION}

The prospect of new colliding-beam accelerators capable of producing collisions of highly stripped high- $Z$ ions, at fixed-target energies per nucleon up to $20 \mathrm{TeV}$ or more, has motivated much interest in lepton-pair production from the QED vacuum. The time-dependent and essentially classical electromagnetic fields ${ }^{1}$ involved in such collisions contain large Fourier components which give rise to sizable leptonpair production ${ }^{2}$ in addition to many other exotic particles 3,4 . In particular, the process of electron-positron production with electron capture is a principal beam-loss mechanism for highly charged ions in a storage ring, and, thus, plays a central role in the design and operation of these machines 5,6 . In this process, the electron is created in a bound state of one of the participant heavy ions (most likely the $1 \mathrm{~s}$ state), thus changing the ion's charge state and causing it to be deflected out of the beam. This topic has been discussed in the review articles in Refs. 7 and 8, and recent experimental results are discussed in Refs. 9 and 10.

There is a long and sometimes controversial ${ }^{5}$ history concerning the use of perturbative methods in studying electromagnetic lepton-pair production; however, reliable perturbative calculations have been used as input into design models for the Relativistic Heavy-ion Collider (RHIC) $)^{2}, 11$. Applying perturbation theory to these processes at high energies and small impact parameters results in probabilities which violate unitarity, and cross sections which violate the Froissart bound $5,2,12$. This evidence, along with the initial nonperturbative studies, suggests that higher-order 
QED effects will be important for extreme relativistic collisions 13,5 . Clearly, large nonperturbative effects in electron-pair production with capture would have important implications for RHIC.

In this paper, I will briefly discuss recent progress in nonperturbative studies of the capture problem. In Section II, we state the Dirac equation for a lepton in the time-dependent external field of a heavy ion which must be solved to compute leptoncapture probabilities. The reader is referred to Refs. 15 and 14 for the details of the formalism. Section IV surveys results from recent applications of coupled-channel and lattice techniques to the lepton-capture problem. We draw conclusions and give an outlook in Section V.

\section{SEMICLASSICAL APPROXIMATION}

We study the electromagnetic production of lepton pairs with capture in a reference frame in which one of the nuclei is at rest, since recoil may be neglected. The target nucleus and the lepton interact via the static Coulomb field, $A_{\mathrm{T}}^{0}$. The only time-dependent interaction, $A_{\mathrm{p}}^{\mu}(t)$, arises from the classical motion of the projectile. Splitting the Dirac Hamiltonian into static and time-dependent parts, we write the Dirac equation for a lepton described by a spinor $\phi(\vec{r}, t)$ coupled to an external, time-dependent electromagnetic field

$$
\left[H_{F}+H_{\mathrm{P}}(t)\right] \phi(\vec{r}, t)=i \frac{\partial}{\partial t} \phi(\vec{r}, t)
$$

where the static Furry Hamiltonian, $H_{\mathrm{F}}$, is given by

$$
H_{\mathrm{F}}=-i \vec{\alpha} \cdot \nabla+\beta-e A_{\mathrm{T}}^{0}
$$

and the time-dependent interaction of the lepton with the projectile is

$$
H_{\mathrm{P}}(t)=e \vec{\alpha} \cdot \vec{A}_{\mathrm{P}}(t)-e A_{\mathrm{P}}^{0}(t)
$$

In Refs. 14 and 15, probabilities for vacuum production of leptons with capture into a bound state $b$ are determined by computing the expectation value of the lepton number operator for the state $b$ with respect to the time-evolved vacuum state. The reader is referred to these references for details of the formalism.

The physics of lepton-pair production is defined by the electromagnetic fields of two particles in relative motion, and these fields enter the Hamiltonian via the dimensionless interaction energy, $\bar{A}^{\mu} \equiv-e A^{\mu}$, between the lepton and the colliding nuclei in Eqs. (2) and (3). For simplicity of discussion, we assume a point-like charge for both the projectile and the target. However, for heavy-lepton production, finitesize effects are importani, and uniform, spherical charge distributions will be used for calculations of these leptons without further discussion. In the target frame, we choose the projectile to move in the $z$-direction, and the reaction plane to be the $y$ - $z$ plane, i.e. the classical trajectory of the projectile is $x_{\mathrm{P}}(t)=0, y_{\mathrm{P}}(t)=b, z_{\mathrm{P}}(t)=\beta_{f} t$. Since the Dirac equation is covariant under a gauge transformation of the electromagnetic potentials, in principle, the gauge may be chosen for convenience in any problem. The most familiar gauge used in problems with electric sources is the Lorentz gauge, defined by the condition $\partial_{\mu} A^{\mu}=0$. The time-dependent electromagnetic interaction 
between the projectile and the lepton in the Lorentz gauge can be generated by a Lorentz-boost of the static Coulomb field. This results in

$$
\begin{aligned}
& \bar{A}_{\mathrm{P}}^{0}\left[r^{\prime}(t)\right]=\frac{-Z_{\mathrm{P} \alpha \gamma_{f}}}{\sqrt{x^{2}+(y-b)^{2}+\gamma_{f}\left(z-\beta_{f} t\right)}} \\
& \bar{A}_{\mathrm{P}}^{3}\left[r^{\prime}(t)\right]=\beta_{f} \bar{A}_{\mathrm{P}}^{0}\left[r^{\prime}(t)\right] \\
& \bar{A}_{\mathrm{P}}^{1}=\bar{A}_{\mathrm{P}}^{2}=0,
\end{aligned}
$$

where $Z_{\mathrm{P}}$ is the atomic number of the projectile, and $\alpha$ is the fine-structure constant. The Lorentz factor in the fixed-target frame is $\gamma_{f}$. The beam energy for a given frame of reference is $E_{\mathrm{kin}}=m_{0} c^{2}(\gamma-1)$, where $\gamma$ denotes the Lorentz factor for the frame of interest. The Lorentz factors for the fixed target and collider frames are related by $\gamma_{f}=2 \gamma_{c}^{2}-1$.

\section{NONPERTURBATIVE CALCULATIONS}

The projectile Hamiltonian represented in the Lorentz gauge is

$$
H_{\mathrm{P}}(\vec{r}, t)=\left(1-\beta_{f} \alpha_{z}\right) \bar{A}_{\mathrm{P}}^{0}\left[r^{\prime}(t)\right]
$$

where $\alpha_{z}$ is the third spin matrix in the Dirac representation. The maximum of the scalar and vector components, which are proportional with proportionality constant $\beta_{f} \approx 1$, scale as $\gamma_{f}$ and their half-width is inversely proportional to $\gamma_{f}$. However, at extreme energies, the probability for capture at a finite impact parameter $b$ is independent of the energy 16,17 . As can be anticipated from the form of Eq. 5, this independence on the energy implies that large cancellations occur between the scalar and vector amplitudes which are troublesome for most approximate solutions ${ }^{16}$. In addition, the spiked nature of the Lorentz-gauge interaction results in the multipole expansion used in the coupled-channel approach having poor convergence properties ${ }^{18}$, and requires extremely fine grid spacing for a faithful representation of the interaction on the lattice. As a result, solving the Dirac equation using the Lorentz-gauge interaction is difficult, and other noncovariant gauge choices have been used in nonperturbative capture calculations. However, good progress has been made by some groups using the Lorentz-gauge interaction. In this section, I will briefly survey recent nonperturbative capture studies, and the choice of gauge when different from the Lorentz gauge.

\section{Coupled-channel Calculations}

In the coupled-channel approach, Eq. (1) is solved by expanding $\phi(\vec{r}, t)$ in the complete, orthonormal set of eigenstates $\chi_{k}(\vec{r}) \exp \left(-i E_{k} t\right)$ of the Furry Hamiltonian, $H_{\mathrm{F}}$. Insertion of the expansion

$$
\phi(\vec{r}, t)=\sum_{k} a_{j k}(t) \chi_{k}(\vec{r}) \exp \left(-i E_{k} t\right)
$$


into Eq. (1) and projection leads to the infinite system of first-order coupled differential equations for the occupation amplitudes $a_{j k}(t)$,

$$
\dot{a}_{j f}(t)=-i \sum_{k} a_{j k}(t)\left\langle\chi_{f}\left|H_{\mathrm{P}}\right| \chi_{k}\right\rangle \exp \left(i\left(E_{f}-E_{k}\right) t\right),
$$

which is equivalent to Eq. (1) 19,18 . In the coupled-channel approach, Eqs. (7) are integrated numerically after the infinite summation is truncated to include a finite number of states. The amplitudes $a_{j f}$ determine the motion of a single lepton during the collision, and, after substitution into Eq. (6), provide the approximate solution to the time-dependent Dirac equation needed to compute the capture probability. The reader is referred to Refs. 18 and 19 for more details.

Recently, coupled-channels calculations with limited basis sets (334 states) have been performed at moderately relativistic fixed-target energies $(1.2$ and $2.0 \mathrm{GeV}$ per nucleon) for $U^{92+}+U^{92+}$ which suggest that perturbative approaches underestimate electron-pair production with capture into the $K$-shell at small impact parameters by a factor of 50 to $100^{19}$. A strong nonperturbative dependence on the projectile charge is also observed. Reference 19 clearly argues the fact that coupled-channel calculations using a truncated basis are not formally gauge invariant, but fail to discuss the degree to which their calculations depend on the gauge choice.

Reference 20 also contains coupled-channel calculations with the ability to use larger basis sets (1700 and 3400 states) and explores the use of noncovariant gauges. In particular, this work reproduces the exact calculations presented in Ref. 19 and demonstrates that the use of a noncovariant gauge results in a change in the capture probability by a factor of two. This work performs large, coupled-channel calculations to predict an upper limit on the electron-capture probabilities at RHIC energies to be a $10 \%$ effect. In related work by this group, the argument is made that the nonperturbative capture cross section should scale with the energy as $\ln \gamma_{f}^{17}$. The significance of this result is that it implies that results of experiments at the lower energies of present-day accelerators may be reasonably extrapolated to the extreme conditions of RHIC.

\section{Lattice Calculations}

In lattice solutions to the Dirac equation, one forsakes a continuous representation of the quantum-state vector and coordinate-space operators in favor of a representation only on a discrete set of spatial lattice points, e.g. $\chi(\vec{r}) \rightarrow \chi_{\alpha, \beta, \gamma}$. Therefore, Dirac spinors become discrete vectors of $4 N_{x} N_{y} N_{z}$ complex numbers, and the Dirac Hamiltonian becomes a matrix of the same rank. Local operators like the electromagnetic interaction become diagonal matrices with the values along the diagonal being simply the value of the interaction at a given lattice point. Nonlocal operators, like coordinate-space derivatives, are represented by matrices which may be banded, as in the finite-difference method, or full, as in basis-spline and spectral methods. In doing this, one reduces the partial-differential equation with specified boundary conditions to a series of linear equations which may be solved using elimination or iterative techniques 24,21 .

For infinitesimal lattice spacing, lattice solutions reproduce the exact, time-dependent solutions of the analytical Dirac equation, which, as stated earlier, are gauge invariant. To insure that solutions are reliable, one must increase the spatial and temporal resolution of the lattice to test for convergence. However, for finite-lattice spacing, 
certain gauges may be more easily represented on the lattice, and different gauges may give different results ${ }^{14}$.

The Lorentz-gauge interaction has been used in capture calculations during central collisions of $\mathrm{U}^{92+}+\mathrm{U}^{92+}$ at $10 \mathrm{GeV}$ per nucleon using a two-dimensional finitedifference lattice approach. Using a very fine grid $(300 \times 600$ points), Ref. 22 reports capture probabilities which are also approximately two-orders of magnitude greater than perturbation theory. The authors state that slight overestimation of the capture probability results from their use of Sommerfeld-Maue analytic continuum states during the calculation of the capture probability, since these states do not form an orthonormal set. Reference 23 extends the coupled-channel calculations, reported in Ref. 19 , to $U^{92+}+U^{92+}$ at $10 \mathrm{GeV}$ per nucleon to obtain good agreement with the finite-difference calculations of Ref. 22.

Reference 14 describes our approach to the lepton-capture problem in which we solve Eq. (1) in three-Cartesian dimensions using lattice-basis-spline techniques. These methods have many advantages over finite-difference techniques, which make an unrestricted solution in three spatial dimensions possible, among which are accuracy, stability, and an undoubled energy spectrum ${ }^{27}$. Lattice calculations for the electroncapture process are more interesting and more difficult than the muon-capture problem. The fundamental difficulty is one of having a number of natural length scales involved in the electron capture problem. These length scales are the nuclear radius, the electron's Compton wavelength, and the size of the heavy atom. (For the muoncapture problem, the muon Compton wavelength and the nuclear radius are the same within a factor of 2.) As a consequence, we have presently deferred an attack on the more nonperturbative and interesting electron-capture problem in favor of the muon-capture problem.

To avoid the stated difficulties of the Lorentz-gauge interaction, we have explored the use of noncovariant gauges. One gauge which is easier to represent on the lattice is the axial gauge, defined by requiring the z-component of the Lorentz-gauge interaction to vanish. The axial gauge has several features which result in a more faithful representation of the interaction on the lattice ${ }^{14}$.

In computing the capture probability, one needs access to the lattice representation of the Coulomb-Dirac continuum states. However, complete diagonalization of the Hamiltonian matrix exceeds current computational capabilities. As a result, we currently approximate the Coulomb-Dirac continuum states using the lattice representation of Dirac plane wave states modified to be orthogonal to the bound states on the lattice ${ }^{14}$. This approximation may be efficiently implemented and is more accurate for use in electron-capture calculations at high energies where Coulomb distortion is a relatively small effect. We note that numerical methods exist to allow extraction of the probabilities for transition to the exact Coulomb distortion continuum states ${ }^{28}$; however, past implementations of these techniques have proved to entail a prohibitively large computational expense.

\section{PRELIMINARY LATTICE-BASIS-SPLINE RESULTS}

In order to gain confidence in our nonperturbative solution of the time-dependent Dirac equation, we seek to reproduce low-order calculations in a perturbative regime. In order to assure that perturbation theory is reasonable, we perform calculations for inuon capture at a collider energy per nucleon of $2 \mathrm{GeV}$ for the system $\mathrm{Au}^{79+}+\mathrm{Au}^{79+}$. At this energy, the maximum frequency in the virtual-photon spectrum of the elec- 


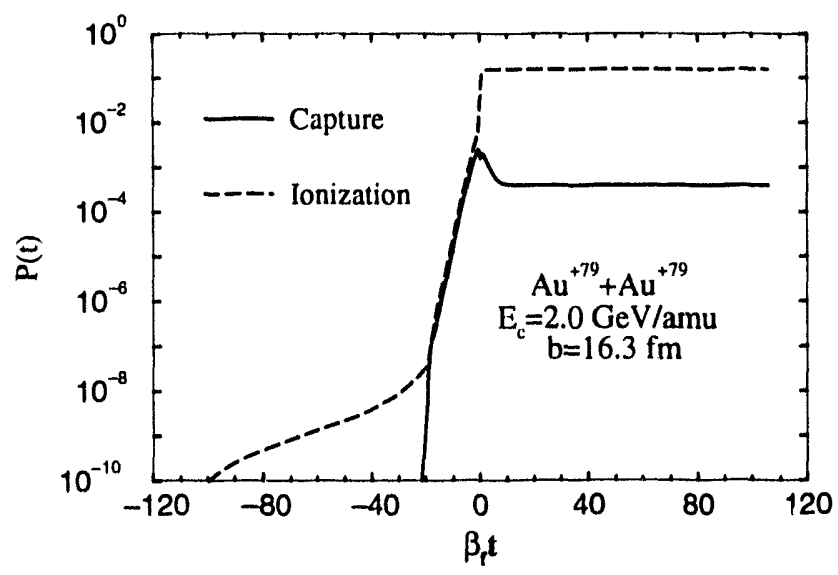

Figure 1: Plotted is the time-dependent probabilities for capture and ionization during collisions of two fully stripped Au nuclei at collider frame energy of $2 \mathrm{GeV}$ per nucleon at an impact parameter of $16.3 \mathrm{fm}$. The time axis is represented by the position along the $z$-axis, $\beta_{f} t$, of the projectile in units of the muon Compton wavelength, $\lambda_{\mu}$.

tromagnetic field is approximately $2 m_{\mu} c^{2}$, the threshold value required to produce a pair. We stress that the calculations presented here should be considered preliminary and no quantitative conclusions should be drawn, as convergence tests with the numerical box size and lattice spacing have not been performed. In addition, as previously mentioned, the approximate continuum states employed for these calculations make quantitative comparison difficult. Nevertheless, calculations with such continuum states are useful, as they can be compared to first-order perturbative calculations performed on the lattice employing the same approximate continuum states. These comparisons provide a stringent test on the dynamics of the nonperturbative solution.

Figure 1 shows the capture and ionization probabilities as a function of time multiplied by the projectile's velocity for a grazing impact parameter of $16.3 \mathrm{fm}$. The ionization probability is approximately 0.1 and the capture probability is approximately $4 \times 10^{-4}$. At no time during the collision is the muon-capture probability greater than $1 \%$. This is consistent with a perturbative process. These numbers highlight another difficulty in performing capture calculations. Sufficient accuracy must be available to extract relatively small capture probabilities out of a time-dependent state dominated by excitation and ionization channels. Figure 2 shows the expectation value of the total Dirac Hamiltonian with respect to the time-dependent Dirac

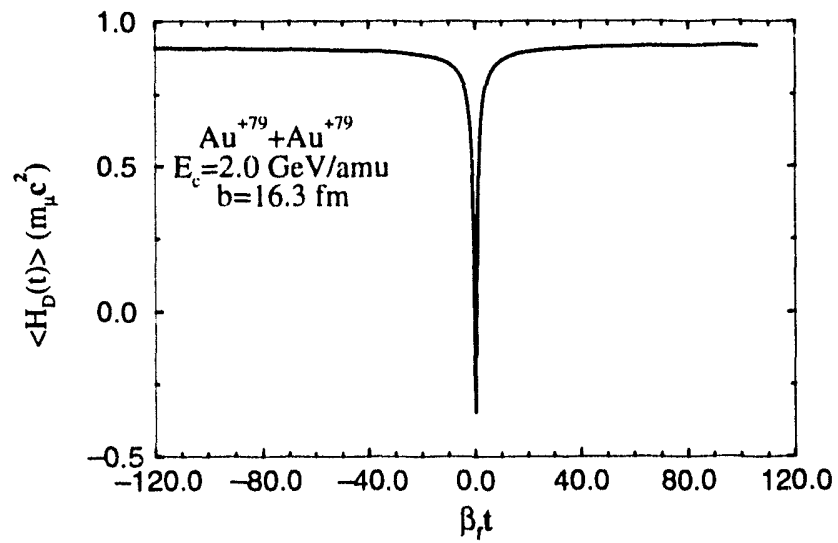

Figure 2: Plotted is the expectation value of the full Dirac Hamiltonian in units of the muon rest mass during the collision described in Fig. 1. 


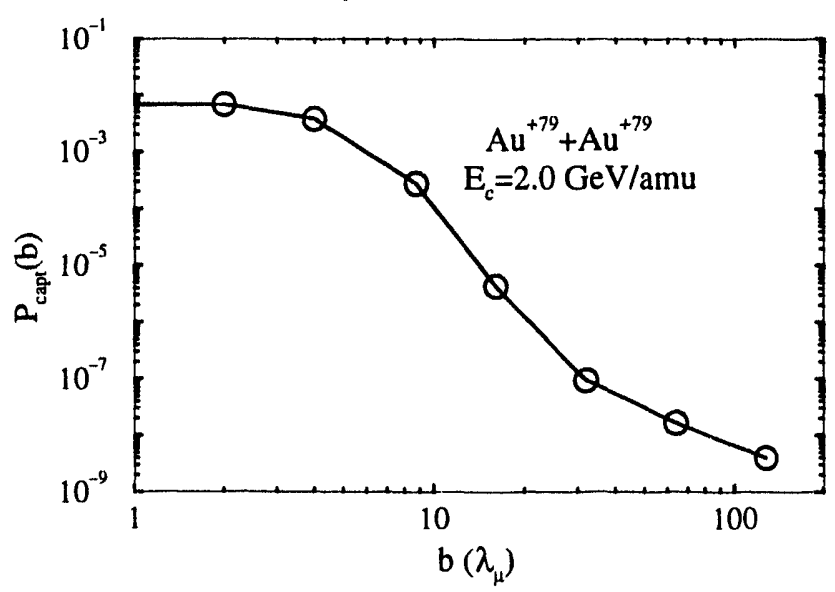

Figure 3: Plotted is the calculated impact-parameter dependence of the muon-capture probability in collisions of $\mathrm{Au}^{92+}+\mathrm{Au}^{92+}$ at a collider energy of $E_{c}=2 \mathrm{GeV}$ per nucleon.

spinor for the same collision as Fig. 1. One sees that the system is excited near the distance of closest approach, then relaxes as time further increases.

In Fig. 3, we show a preliminary impact parameter dependence for the muoncapture probabilities at collider energies per nucleon of $2 \mathrm{GeV}$. Notice initially the probabilities fall exponentially with $b$ as expected from perturbation theory for the large impact parameters. However, as the capture probability becomes very small, one observes a change in the $b$-dependence to a power law. This this power-law dependence is undesirable and results from finite-lattice sizes effecting the small probabilities.

\section{COMMENTS AND OUTLOOK}

Recent progress has been made in the theoretical understanding of nonperturbative lepton-pair production with capture as is discussed in this short paper. Most exciting is that pioneering experiments have recently been reported 9,10 , and the second generation of experiments are planned for the near future 25,26 .

As a general comment, all nonperturbative approaches mentioned in this paper sacrifice, to some extent, the gauge invariance of the semiclassical theory used to describe the capture problem. The extent to which gauge invariance is violated in practical calculations gives a measure of the reliability of these calculations and should be reported along with quantitative predictions.

Fundamental improvements have been achieved recently in the application of lattice techniques to the Dirac equation 27 , and more progress is anticipated. Specifically, our time-dependent Dirac code currently running on the Intel iPSC/860 hypercube parallel computer will be ported to the Intel's next generation of massively parallel computers, the Paragon XP/S series. This machine will provide the computing power necessary to demonstrate numerical convergence in our large calculations. In addition, a new and efficient algorithm for accurate energy analysis of the time-dependent Dirac state will be implemented and tested ${ }^{28}$. The combination of these two readily accessible improvements will allow us to make accurate predictions for muon-pair production with capture at collision energies accessible to the present-day heavy-ion accelerators. 


\section{ACKNOWLEDGEMENTS}

This research was sponsored in part by the U.S. Department of Energy (DOE) under contract No. DE-AC05-840R21400 managed by Martin Marietta Energy Systems, Inc., under contract No. DE-FG05-87ER40376 with Vanderbilt University, and under the High Performance Computing and Communications Program (HPCC), as a Grand Challenge titled the Quantum Structure of Matter. The numerical calculations were carried out on the Intel iPSC/860 hypercube multicomputer at the Oak Ridge National Laboratory, the CRAY-2 supercomputers at the National Energy Research Supercomputer Center (NERSC) at Lawrence Livermore National Laboratory, and the National Center for Supercomputing Applications (NCSA) in Illinois.

\section{REFERENCES}

1. J.-S. Wu, C. Bottcher, M. R. Strayer, Phys. Lett. B252, 37 (1993).

2. C. Bottcher and M. R. Strayer, Phys. Rev. D 39, 1330 (1989).

3. J.-S. Wu, C. Bottcher, M. R. Strayer, and A. K. Kerman, Ann. Phys. 210, 402 (1991).

4. G. Soff, this conference.

5. Can RHIC be used to test QED?, Brookhaven National Laboratory Workshop Proceedings, Upton, New York, BNL-52247, April 1990.

6. M. J. Rhoades-Brown, C. Bottcher, and M. R. Strayer, Nucl. Instrum. Meth. B43 (1989) 301.

7. C. A. Bertulani and G. Baur, Phys. Rep. 163, 299 (1988).

8. J. Eichler, Phys. Rep. 193, 167 (1990).

9. A. Belkacem, H. Gould, B. Feinberg, R. Bossingham, and W. E. Meyerhof, Phys. Rev. Lett. 71, 1514 (1993).

10. A. Westphal and Y. D. He, Phys. Rev. Lett. 711160 (1993).

11. M. J. Rhoades-Brown, T. Ludlam, J.-S. Wu, C. Bottcher, and M. R. Strayer, Fourth Workshop on Experiments and Detectors for a Relativistic Heavy-ion Collider, Brookhaven National Laboratory Report, BNL 52262, 325 (July 1990).

12. M. Froissart, Phys. Rev. 123, 1053 (1961).

13. C. Bottcher and M. R. Strayer, Nucl. Inst. and Meth. B31, 122 (1988).

14. J. C. Wells, V. E. Oberacker, A. S. Umar, C. Bottcher, M. R. Strayer, J.-S. Wu, and G. Plunien, Phys. Rev. A 45 (1992) 6296.

15. V. E. Oberacker, J. C. Wells, A. S. Umar, and M. R. Strayer, contribution to this school. 
16. P. A. Amundsen and K. Aashamar, J. Phys. B. 14, 4047 (1981).

17. A. J. Baltz, M. J. Rhoades-Brown, and J. Weneser, Phys. Rev. A 44, 5569 (1991).

18. W. Scheid, contribution to this school.

19. K. Rumrich, G. Soff, and W. Greiner, Phys. Rev. A 47, 215 (1993); K. Rumrich, K. Momberger, G. Soff, W. Greiner, N. Grün, and W. Scheid, Phys. Rev. Lett. 66, 2613 (1991).

20. A. J. Baltz, M. J. Rhoades-Brown, and J. Weneser, Phys. Rev. A 47, 3444 (1993).

21. J. C. Wells, V. E. Oberacker, A. S. Umar, C. Bottcher, M. R. Strayer, J.-S. Wu, J. Drake, and R. Flanery, Int. J. Mod. Phys. C 4 (1993) 459.

22. J. Thiel, A. Bunker, K. Momberger, N. Grün, and W. Scheid, Phys. Rev. A 46, 2607 (1992).

23. J. Thiel, K. Momberger, N. Grün, and W. Scheid, Proceedings of the International Symposium on Nuclear Physics of our Times, November 17-21, 1992, Sanibel Island, Florida.

24. U. Becker, N. Grün, and W. Scheid, J. Phys. B 16, 1967 (1983).

25. Experiment WA99, approved by CERN SPS Program Committee, March, 1993. Measurements of Pair Production and Electron Capture from the Continuum in Heavy Particle Collisions, Oak Rdige National Laboratory, Manne Siegbahn Institute of Physics, Univ. of Aarhus, and Univ. of Lund.

26. H. Gould, private communication.

27. J. C. Wells, V. E. Oberacker, M. R. Strayer, and A. S. Umar, Oak Ridge National Laboratory Preprint, ORNL/CCIP/93/12, August, 1993.

28. C. Bottcher, M. R. Strayer, A. S. Umar, and V. E. Oberacker, Phys. Rev. C 37, 2487 (1988). 

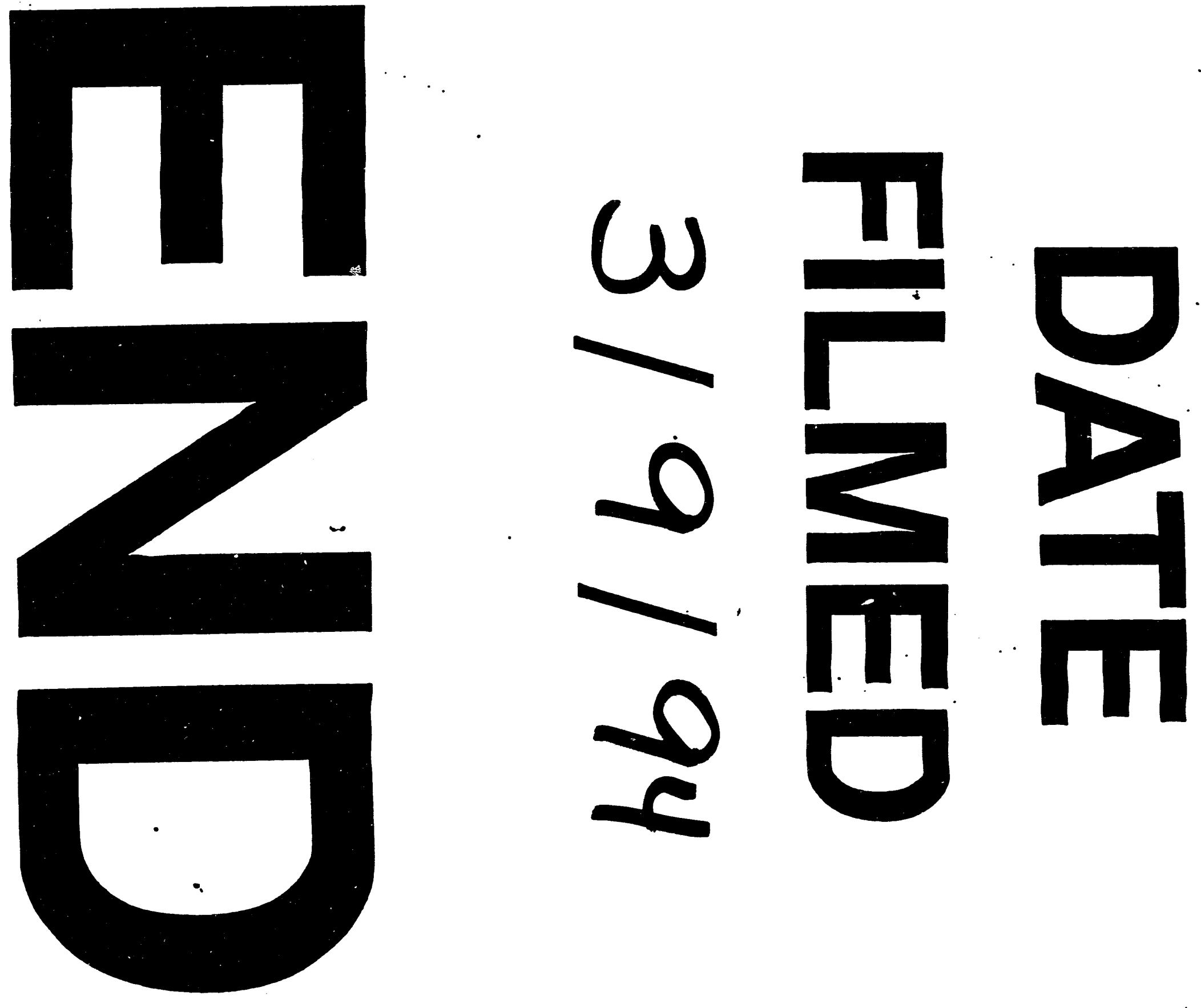